\title{
THERE ARE NO PROPER TOPOLOGICAL HYPERBOLIC HOMOCLINIC CLASSES FOR AREA-PRESERVING MAPS
}

\author{
MÁRIO BESSA AND MARIA JOANA TORRES
}

\begin{abstract}
We begin by defining a homoclinic class for homeomorphisms. Then we prove that if a topological homoclinic class $\Lambda$ associated to an area-preserving homeomorphism $f$ on a surface $M$ is topologically hyperbolic (i.e. has the shadowing and expansiveness properties), then $\Lambda=M$ and $f$ is an Anosov homeomorphism.
\end{abstract}

MSC 2010: primary 37E30; 54H20; secondary 37C50.

Keywords: Shadowing, expansiveness, topological dynamics, homoclinic classes.

\section{INTRODUCTION}

Let $f: M \rightarrow M$ be a diffeomorphism defined on a closed and connected Riemannian manifold $M$. A compact and $f$-invariant set $\Lambda \subset M$ is called a hyperbolic set if the tangent bundle over $\Lambda$ can be decomposed as a direct sum of two sub-bundles both invariant by the tangent map $D f$ that are uniformly contracted under, respectively, forward and backward iterations (see e.g. [9] for full details). When such a hyperbolic set $\Lambda$ is given by the orbit of a periodic point, the point is called a hyperbolic periodic point. The concept of hyperbolicity played a fundamental role in the development of the stability theory of dynamical systems (see e.g. [9, 10]).

We recall that a nonwandering point $x \in M$ is a point such that any neighbourhood $\mathcal{U}$ of $x$ contains points for which some forward iterate is in $\mathcal{U}$. If the set of nonwandering points is the closure of the set of hyperbolic periodic points and furthermore it is a hyperbolic set, then Smale's spectral decomposition theorem ([10]) assures that the set of nonwandering points can be partitioned into a finite number of compact blocks exhibiting a dense orbit, called basic blocks. When there is a single piece in this decomposition, $f$ is called an Anosov map.

In an attempt to generalize the hyperbolic basic sets which played a central role in Smale's spectral decomposition, Newhouse introduced, in the early seventies, the concept of a homoclinic class (see [5]). A homoclinic class is defined as the closure of the set of transversal intersections of the stable and unstable invariant manifolds of a hyperbolic periodic saddle of a diffeomorphism $f$. Homoclinic classes are $f$-invariant and display a dense orbit of $f$ in the homoclinic class. Yet the hyperbolicity of the periodic saddle, which gives origin to the homoclinic class, is not enough to spread hyperbolicity to the whole homoclinic class. Indeed, it is well-known that homoclinic classes may fail to be uniformly hyperbolic.

In the particular case when $M$ is a surface and $f$ is area-preserving, Newhouse proved in the mid eighties [6] a simple but elegant result. He proved that $f$ cannot support proper uniformly hyperbolic homoclinic classes. In other words, uniformly hyperbolic homoclinic classes must be the whole manifold, therefore concluding that $f$ is an Anosov map. 
In the present paper we intend to reconfigure Newhouse's theorem by allowing one to consider maps that are not diffeomorphisms. Two problems arise: the pointwise hyperbolicity (a homoclinic class foreshadows the need for a hyperbolic periodic saddle point) and set hyperbolicity (the hypothesis that a homoclinic class is hyperbolic).

Clearly, we first need to seek vestiges of hyperbolicity in non differentiable contexts and only then try to conjecture what could be the topological counterpart of Newhouse's theorem. Our proposal is to replace the hyperbolicity by two properties with topological flavor - shadowing and expansiveness. In $\$ 2$ we fully describe these 'topological hyperbolic sets' in terms of dynamically defined invariant manifolds, canonical coordinates and local product structure which are of utmost importance when we realign Newhouse's strategy. In $\S 3$ we define topological homoclinic classes readapted to a type of topological transversality and obtain the Birkhoff-Smale theorem in this non differentiable setting.

For diffeomorphisms it is well-known that periodic points are dense in the whole homoclinic class. However, we do now know how to prove this property for homeomorphisms (see Remark 3.1). Fortunately, we are considering hyperbolic topological homoclinic classes which is enough to obtain that periodic points are dense in the whole homoclinic class (cf. Proposition 2.6).

Like Newhouse, who was inspired by a uniformly hyperbolic basic set to consider the closure of transversal intersections of stable/unstable manifolds of a hyperbolic periodic saddle, here we got inspired by a set displaying shadowing and expansiveness (topological set hyperbolicity) to consider the closure of topological transversal intersections of stable/unstable manifolds of a periodic point displaying shadowing and expansiveness (pointwise hyperbolicity).

We hope that our definition of topological homoclinic class can be useful in several aspects of topological dynamics. Finally, in $\S 4$ we prove the next:

Theorem 1. Let $M$ be a surface, $f: M \rightarrow M$ be an area-preserving homeomorphism and let $\Lambda \subseteq M$ be a topological homoclinic class of $f$. If $f$ has the shadowing property on $\Lambda$ and is expansive on $\Lambda$, then $f$ is an Anosov homeomorphism (i.e., $f$ has the shadowing property on $M$ and is expansive on $M$ ).

It is interesting to notice that in $[4,3]$ it was proved that there are no expansive homeomorphisms on the two-dimensional sphere $\mathbb{S}^{2}$. This result along with Theorem 1 allows us to conclude that $\mathbb{S}^{2}$ does not support topological homoclinic classes with shadowing and expansiveness associated to an area-preserving homeomorphism.

Throughout the article we assume that $M$ is a closed, connected Riemannian manifold, $d$ is the distance on $M$ induced by the Riemannian structure and $\lambda$ is the Lebesgue measure on $M$ associated to a volume form on $M$. Despite the fact that our main result is about surfaces some results are stated and proved for manifolds on dimension $\geq 2$ for eventual future use.

\section{TOPOLOGICAL HYPERBOLICITY}

2.1. Hyperbolic homeomorphisms. Let $f: M \rightarrow M$ be a homeomorphism. Given $\delta>0$, a sequence of points $\left\{x_{i}\right\}_{i \in \mathbb{Z}} \subset M$ is called a $\delta$-pseudo-orbit of $f$ if $d\left(f\left(x_{i}\right), x_{i+1}\right)<\delta$ for all $i \in \mathbb{Z}$. Let $\Lambda \subseteq M$ be a closed $f$-invariant set (i.e., $f(\Lambda)=\Lambda$ ). We say that $f$ has the shadowing property on $\Lambda$ if for every $\epsilon>0$ there is $\delta>0$ such that for any $\delta$-pseudo-orbit $\left\{x_{i}\right\}_{i \in \mathbb{Z}} \subset \Lambda$ of $f$ there is a point $z \in \Lambda$ such that $d\left(f^{i}(z), x_{i}\right)<\epsilon$ for all $i \in \mathbb{Z}$. When $\Lambda=M, f$ is said to have the shadowing property. 
A homeomorphism $f$ is called expansive on $\Lambda$ (see $[9, \S 7])$ if there is $e>0$, called an expansive constant, such that for all $x \in \Lambda$ and $y \in M$ if we have $d\left(f^{n}(x), f^{n}(y)\right) \leq e$ for all $n \in \mathbb{Z}$, then $x=y$. When $\Lambda=M, f$ is simply said to be expansive.

A homeomorphism $f$ is called an Anosov homeomorphism if it has the shadowing property and is expansive (see $[1, \S 11.3]$ ). We shall say that $f$ is a hyperbolic homeomorphism on $\Lambda$ if it has the shadowing property on $\Lambda$ and is expansive on $\Lambda$.

2.2. Invariant sets, shadowing and expansiveness. In this section we obtain some useful local results to be used in the sequel. Let $f: M \rightarrow M$ be a homeomorphism and let $\Lambda \subseteq M$ be a closed and $f$-invariant set. Given $x \in M$ and $\epsilon>0$, the local stable and local unstable set of $x$ are defined, respectively, by

$$
W_{\epsilon}^{s}(x)=\left\{y \in M: d\left(f^{n}(x), f^{n}(y)\right) \leq \epsilon, \text { for } n \geq 0\right\}
$$

and

$$
W_{\epsilon}^{u}(x)=\left\{y \in M: d\left(f^{-n}(x), f^{-n}(y)\right) \leq \epsilon, \text { for } n \geq 0\right\} .
$$

Under the expansiveness hypothesis on $\Lambda$, stable and unstable sets of points $x \in \Lambda$ are dynamically defined, i.e., the following result holds.

Proposition 2.1. Let $e>0$. The homeomorphism $f$ is expansive on $\Lambda$ with expansive constant $e$ if and only iffor all $\tilde{e}>0$ there exists $N>0$ such that for all $x \in \Lambda$ and all $n \geq N$ we have

$$
f^{n}\left(W_{e}^{s}(x)\right) \subset W_{\tilde{e}}^{s}\left(f^{n}(x)\right) \text { and } f^{-n}\left(W_{e}^{u}(x)\right) \subset W_{\tilde{e}}^{u}\left(f^{-n}(x)\right) .
$$

Proof. Suppose that $f$ is expansive on $\Lambda$ with expansive constant $e$, and suppose, for a contradiction, that there exist sequences $x_{n} \in \Lambda$ and $y_{n} \in M, n \in \mathbb{N}$, such that $y_{n} \in W_{e}^{s}\left(x_{n}\right)$ and $d\left(f^{n}\left(x_{n}\right), f^{n}\left(y_{n}\right)\right)>\tilde{e}$. Since $y_{n} \in W_{e}^{s}\left(x_{n}\right)$, we have that $d\left(f^{m} \circ f^{n}\left(x_{n}\right), f^{m} \circ f^{n}\left(y_{n}\right)\right) \leq e$ for all $m \geq-n$. Taking subsequences we may assume that there exists $x \in \Lambda$ and $y \in M$ such that $\lim _{n} f^{n}\left(x_{n}\right)=x$ and $\lim _{n} f^{n}\left(y_{n}\right)=y$. Hence, $d\left(f^{m}(x), f^{m}(y)\right) \leq e$ for all $m \in \mathbb{Z}$. Moreover, $d(x, y)=\lim _{n} d\left(f^{n}\left(x_{n}\right), f^{n}\left(y_{n}\right)\right) \geq \tilde{e}$. This contradicts the expansiveness on $\Lambda$.

Conversely, let $x \in \Lambda$ and $y \in M$ be such that $d\left(f^{n}(x), f^{n}(y)\right) \leq e$ for all $n \in \mathbb{Z}$. For all $n \geq 0$ we have $f^{-n}(y) \in W_{e}^{s}\left(f^{-n}(x)\right)$. Then, for any $\tilde{e}>0$ we have $y \in W_{\tilde{e}}^{s}(x)$ by the first inclusion of (2.1) and hence $x=y$.

The stable and unstable sets of $x \in M$ are defined, respectively, by

$$
W^{s}(x)=\left\{y \in M: \lim _{n \rightarrow+\infty} d\left(f^{n}(x), f^{n}(y)\right)=0\right\}
$$

and

$$
W^{u}(x)=\left\{y \in M: \lim _{n \rightarrow+\infty} d\left(f^{-n}(x), f^{-n}(y)\right)=0\right\} .
$$

Proposition 2.2. If $f$ is expansive on $\Lambda$ with expansive constant e and $\epsilon \in(0, e)$, then for all $x \in \Lambda$ :

$$
W^{s}(x)=\bigcup_{n \geq 0} f^{-n}\left(W_{\epsilon}^{s}\left(f^{n}(x)\right)\right) \text { and } W^{u}(x)=\bigcup_{n \geq 0} f^{n}\left(W_{\epsilon}^{u}\left(f^{-n}(x)\right)\right) .
$$


Proof. Let $y \in \cup_{n \geq 0} f^{-n} W_{\epsilon}^{s}\left(f^{n}(x)\right)$. There exists $n \geq 0$ such that $f^{n}(y) \in W_{\epsilon}^{s}\left(f^{n}(x)\right)$. By Proposition 2.1, for all $\tilde{e}>0$ there exists $N>0$ such that for all $m \geq N$

$$
f^{m+n}(y) \in f^{m} W_{\epsilon}^{s}\left(f^{n}(x)\right) \subset W_{\tilde{e}}^{s}\left(f^{m+n}(x)\right) .
$$

Hence, $d\left(f^{m+n}(y), f^{m+n}(x)\right) \leq \tilde{e}$ for all $m \geq N$ and, consequently, $y \in W^{s}(x)$.

Conversely, let $y \in W^{s}(x)$. Given $\epsilon>0$ there exists $N \geq 0$ such that $d\left(f^{n}(x), f^{n}(y)\right) \leq \epsilon$ for $n \geq N$. Consequently, $d\left(f^{i} \circ f^{N}(x), f^{i} \circ f^{N}(y)\right) \leq \epsilon$ for all $i \geq 0$, i.e., $f^{N}(y) \in W_{\epsilon}^{s}\left(f^{N}(x)\right)$. Hence,

$$
y \in f^{-N} W_{\epsilon}^{s}\left(f^{N}(x)\right) \subset \cup_{n \geq 0} f^{-n} W_{\epsilon}^{s}\left(f^{n}(x)\right) .
$$

The second equality in (2.2) can be proved analogously.

We say that $f$ has canonical coordinates on $\Lambda$ if for each $\epsilon>0$ there exists $\delta>0$ such that if $x, y \in \Lambda$ and $d(x, y)<\delta$, then $W_{\epsilon}^{s}(x) \cap W_{\epsilon}^{u}(y) \cap \Lambda \neq \emptyset$.

Lemma 2.3. If $f$ has the shadowing property on $\Lambda$, then $f$ has canonical coordinates on $\Lambda$.

Proof. Given $\epsilon>0$, let $\delta>0$ be given by the shadowing property on $\Lambda$. Let us be given any $x, y \in \Lambda$ such that $d(x, y)<\delta$. Take $x_{i}=f^{i}(x)$ for $i \geq 0$ and $x_{i}=f^{i}(y)$ for $i<0$. Clearly, $\left\{x_{i}\right\}_{i \in \mathbb{Z}} \subset \Lambda$ is a $\delta$-pseudo orbit of $f$. By the shadowing property on $\Lambda$ there exists $z \in \Lambda$ such that $d\left(f^{i}(z), x_{i}\right)<\epsilon$ for all $i \in \mathbb{Z}$. Thus, $d\left(f^{i}(z), f^{i}(x)\right)<\epsilon$ for all $i \geq 0$ and $d\left(f^{i}(z), f^{i}(y)\right)<\epsilon$ for all $i<0$. Hence, $z \in W_{\epsilon}^{s}(x)$ and $z \in W_{\epsilon}^{u}(y)$.

Lemma 2.4. If $f$ is an expanding homeomorphism on $\Lambda$ with expansive constant $e$, then for each $0<\epsilon \leq e / 2$ there exists $\delta>0$ such that if $x, y \in \Lambda$ and $d(x, y)<\delta$, then there is at most one point of intersection of $W_{\epsilon}^{s}(x)$ and $W_{\epsilon}^{u}(x)$.

Proof. Let $z \in W_{\epsilon}^{s}(x) \cap W_{\epsilon}^{u}(y)$. Suppose, by contradiction, that there exists $w \in M, w \neq z$, such that $w \in W_{\epsilon}^{s}(x) \cap W_{\epsilon}^{u}(y)$. Then, for all $n \geq 0, d\left(f^{n}(x), f^{n}(z)\right) \leq \epsilon, d\left(f^{n}(x), f^{n}(w)\right) \leq \epsilon$, $d\left(f^{-n}(y), f^{-n}(z)\right) \leq \epsilon$, and $d\left(f^{-n}(y), f^{-n}(w)\right) \leq \epsilon$. This implies that

$$
d\left(f^{n}(z), f^{n}(w)\right) \leq 2 \epsilon,
$$

for all $n \in \mathbb{Z}$, which contradicts the expansiveness on $\Lambda$.

As a consequence of Lemmas 2.3 and 2.4 we have:

Corollary 2.5. If $f$ is a hyperbolic homeomorphism on $\Lambda$ with expansive constant $e$, then for each $0<\epsilon \leq e / 2$ there exists $\delta>0$ such that if $x, y \in \Lambda$ and $d(x, y)<\delta$, then

$$
W_{\epsilon}^{s}(x) \cap W_{\epsilon}^{u}(y)=\{\text { one point }\} \subset \Lambda .
$$

Remark 2.1. We say that a 1-dimensional set $W \subset M$ is a topological manifold if every $x \in W$ has a neighborhood homeomorphic to $\mathbb{R}$. It should be enlightening to say that even with expansiveness we can have several 'prongs' as invariant sets and thus stable (and unstable) sets are not topological manifolds. In Figure 2 we can have expansiveness, nevertheless a set $\Lambda$ including a ball around $p$ cannot have canonical coordinates. Actually, there exist points $x, y \in \Lambda$ arbitrarily close but still without any intersection between the local stable set of $x$ with the local unstable set 
of y. Shadowing implies no n-prongs structure for $n>2$. Expansivity implies no 1-prong structure (cf. Figure 1). Therefore, when we put together shadowing and expansiveness there can only be a 2-prongs structure (Figure 3).

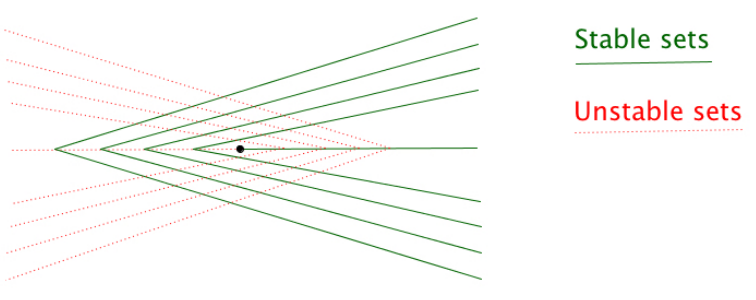

Figure 1. Example of a 1-prong structure. In this case local stable and local unstable sets intersect in more than one point contradicting expansivity (cf. Lemma 2.4).

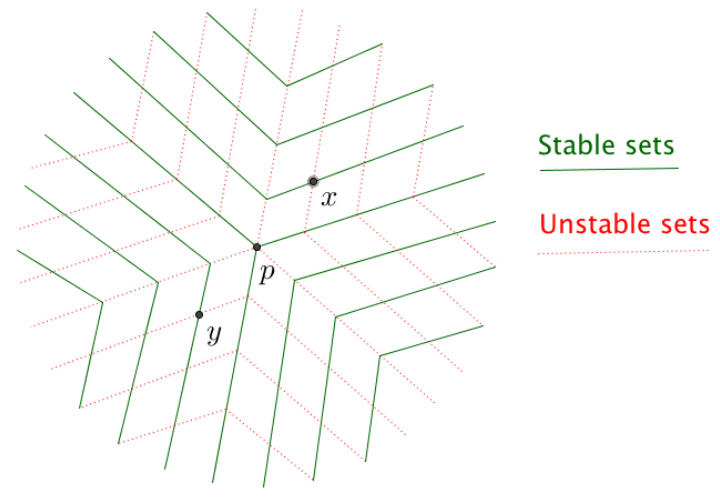

Figure 2. Example of a 3-prongs structure. In this case local stable and local unstable sets do not intersect contradicting the shadowing property (cf. Lemma 2.3).

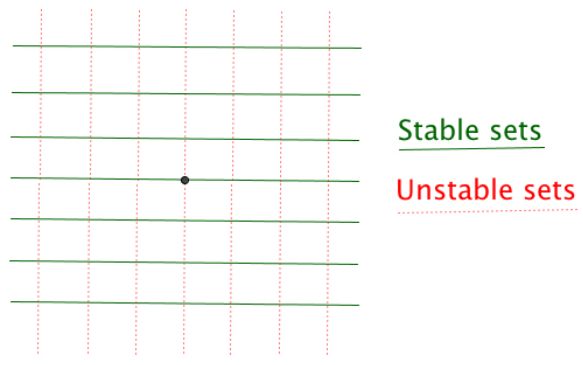

FIGURE 3. Example of 2-prongs structure which is compatible with being a $C^{0}$ manifold.

The next result shows that hyperbolic homeomorphisms on $\Lambda$ behave somehow like basic pieces of classical hyperbolic dynamics theory (cf. [9]). 
Proposition 2.6. Let $f: M \rightarrow M$ be a volume-preserving homeomorphism which is hyperbolic on $\Lambda$. Then, $\overline{\operatorname{Per}(f) \cap \Lambda}=\Lambda$.

Proof. Let $x \in \Lambda$. Since $f$ is volume-preserving, by Poincaré recurrence theorem, almost every point in $\Lambda$ is recurrent and, therefore, $x$ is a non-wandering point. Hence, it is easy to deduce that for every $\delta>0$, there exists a $\delta$-chain from $x$ to itself, i.e., there exists a finite $\delta$-pseudo-orbit $\left\{x_{i}\right\}_{i=0}^{n} \subset \Lambda$ such that $x=x_{0}=x_{n}, n>0$.

Let $e$ be the expansive constant of $f$, let $0<\epsilon \leq e / 2$ be fixed and let $\delta>0$ be given by the shadowing property on $\Lambda$. Let $\left\{x_{i}\right\}_{i=0}^{N} \subset \Lambda$ be a $\delta$-chain from $x$ to itself. The sequence $\left\{\tilde{x}_{i}\right\}_{i \in \mathbb{Z}}$ defined by $\tilde{x}_{i}=x_{i}$ if $n \equiv i(\bmod \mathrm{N})$ is a $\delta$-pseudo orbit. Therefore, it is $\epsilon$-shadowed by some point $z \in \Lambda$ and, clearly, also by $f^{N}(z) \in \Lambda$. Applying the triangle inequality, we have that, $d\left(f^{n}(z), f^{n+N}(z)\right) \leq 2 \epsilon \leq e$, for all $n \in \mathbb{Z}$. Hence, by the expansive property on $\Lambda$, we obtain that $z=f^{N}(z)$. Thus, $z \in \Lambda$ is a periodic point such that $d(z, x)=d\left(z, x_{0}\right) \leq \epsilon$.

2.3. Local product structure. Given $\epsilon>0$, let $\Delta(\epsilon):=\{(x, y): x, y \in \Lambda$ and $d(x, y) \leq \epsilon\}$. We say that a homeomorphism $f$ has a local product structure (LPS) on $\Lambda$ if

(A) there are $\delta_{0}>0$ and a continuous function

$$
\begin{array}{rllc}
{[\cdot, \cdot]:} & \Delta\left(\delta_{0}\right) & \longrightarrow \Lambda \\
(x, y) & \longmapsto[x, y]
\end{array}
$$

such that for all $x, y, z \in \Lambda$ we have

$$
[x, x]=x,[[x, y], z]=[x, z],[x,[y, z]]=[x, z] \text { and } f([x, y])=[f(x), f(y)],
$$

whenever defined.

(B) There exist $\delta_{1} \in\left(0, \delta_{0} / 2\right)$ and $\rho \in\left(0, \delta_{1}\right)$ such that for all $x \in \Lambda$ the following three conditions hold:

(i) denoting $V_{\delta_{1}}^{\sigma}(x):=\left\{y \in W_{\delta_{0}}^{\sigma}(x) \cap \Lambda: d(x, y)<\delta_{1}\right\}(\sigma \in\{s, u\})$ we have that $\left[V_{\delta_{1}}^{u}(x), V_{\delta_{1}}^{s}(x)\right]$ is an open set of $\Lambda$ with diameter less than $\delta_{0}$;

(ii) $[\cdot, \cdot]: V_{\delta_{1}}^{u}(x) \times V_{\delta_{1}}^{s}(x) \rightarrow\left[V_{\delta_{1}}^{u}(x), V_{\delta_{1}}^{s}(x)\right]$ is a homeomorphism and

(iii) $\left[V_{\delta_{1}}^{u}(x), V_{\delta_{1}}^{s}(x)\right] \supset\{y \in \Lambda: d(x, y) \leq \rho\}$.

When $f$ is a hyperbolic homeomorphism on $\Lambda$ we shall set $[x, y]:=W_{\eta}^{s}(x) \cap W_{\eta}^{u}(y)$, where $\eta=\frac{e}{4}$ and $e$ is an expansive constant for $f$ (and $x$ is near $y$ ).

Lemma 2.7. If $f$ is a hyperbolic homeomorphism on $\Lambda$, then $f$ has a local product structure on $\Lambda$.

Proof. We borrow ideas from [2, Theorem 5.6] and next we adapt it to the local case. Let $e>0$ be an expansive constant for $f$ and fix $\eta=\frac{e}{4}$. By Corollary 2.5, there exists $0<\delta_{0}<\eta$ such that $W_{\eta}^{s}(x) \cap W_{\eta}^{u}(y)=\{$ one point $\} \subset \Lambda$ for $x, y \in \Lambda$ with $d(x, y) \leq \delta_{0}$. We define the map $[\cdot, \cdot]: \Delta\left(\delta_{0}\right) \rightarrow$ $\Lambda$ by $[x, y]:=W_{\eta}^{s}(x) \cap W_{\eta}^{u}(y)$, for $(x, y) \in \Delta\left(\delta_{0}\right)$.

The map $[\cdot, \cdot]$ is continuous. Indeed, let $\left\{\left(x_{n}, y_{n}\right)\right\} \subset \Delta\left(\delta_{0}\right)$ be a sequence that converges to $(x, y) \in \Delta\left(\delta_{0}\right)$. Let $z_{n}=\left[x_{n}, y_{n}\right]$. Since $\Lambda$ is compact, taking subsequences, we may assume that $\left(z_{n}\right)_{n}$ converges to $z \in \Lambda$. Since $z_{n} \in W_{\eta}^{s}\left(x_{n}\right)$, we have that $d\left(f^{i}\left(x_{n}\right), f^{i}\left(z_{n}\right)\right) \leq \eta(i \geq 0)$. Hence, $d\left(f^{i}(x), f^{i}(z)\right) \leq \eta$ and, therefore, $z \in W_{\eta}^{s}(x)$. Analogously, $z \in W_{\eta}^{u}(y)$. Thus $z=[x, y]$ which shows that $\left(z_{n}\right)_{n}=\left(\left[x_{n}, y_{n}\right]\right)_{n}$ converges to $[x, y]$.

Clearly, $[x, x]=x$ for all $x \in \Lambda$. Since $[x, y] \in W_{\eta}^{s}(x)$, we have that $[[x, y], z] \in W_{2 \eta}^{s}(x) \cap W_{\eta}^{u}(z)$. Hence, by expansivity on $\Lambda,[[x, y], z]=[x, z]$. Analogously, $[x,[y, z]]=[x, z]$. By uniform 
continuity of $f$ on $\Lambda$, it is easy to conclude that $f[x, y]=[f(x), f(y)]$. All together this proves (A) in the definition of LPS on $\Lambda$.

We shall now prove (B) (i)-(iii) in the definition of LPS on $\Lambda$. We define a map $g_{1}: \Lambda \times$ $\Delta\left(\delta_{0}\right) \rightarrow \mathbb{R}$ by $g_{1}(x,(y, z)):=d(x,[y, z])$, for $x \in \Lambda$ and $(y, z) \in \Delta\left(\delta_{0}\right)$. Clearly, $g_{1}$ is continuous and $g_{1}(x,(x, x))=0$. Since $g_{1}$ is uniformly continuous, there exists $\delta_{1} \in\left(0, \delta_{0} / 2\right)$ such that $\operatorname{diam}\{x, y, z\}<2 \delta_{1}$ implies $d(x,[y, z])<\delta_{0} / 3$. Therefore, given $(y, z) \in V_{\delta_{1}}^{u}(x) \times V_{\delta_{1}}^{s}(x)$, we have that $d(x,[y, z])<\delta_{0} / 3$. Take $w_{1}, w_{2} \in\left[V_{\delta_{1}}^{u}(x), V_{\delta_{1}}^{s}(x)\right]$. Then there exists $y_{1}, y_{2} \in V_{\delta_{1}}^{u}(x)$ and $z_{1}, z_{2} \in$ $V_{\delta_{1}}^{s}(x)$ such that $w_{1}=\left[y_{1}, z_{1}\right]$ and $w_{2}=\left[y_{2}, z_{2}\right]$. We have that $d\left(w_{1}, w_{2}\right)=d\left(\left[y_{1}, z_{1}\right],\left[y_{2}, z_{2}\right]\right) \leq$ $d\left(x,\left[y_{1}, z_{1}\right]\right)+d\left(x,\left[y_{2}, z_{2}\right]\right) \leq \delta_{0} / 3+\delta_{0} / 3<\delta_{0}$. Hence, the diameter of $\left[V_{\delta_{1}}^{u}(x), V_{\delta_{1}}^{s}(x)\right]$ is smaller than $\delta_{0}$. To show that $\left[V_{\delta_{1}}^{u}(x), V_{\delta_{1}}^{s}(x)\right]$ is open in $\Lambda$, let $w \in\left[V_{\delta_{1}}^{u}(x), V_{\delta_{1}}^{s}(x)\right]$. Then there exists $y \in V_{\delta_{1}}^{u}(x)$ and $z \in V_{\delta_{1}}^{s}(x)$ such that $w=[y, z]$. Since $\operatorname{diam}\{x, y, z\}<2 \delta_{1}$ we have that $d(x, w)<\delta_{0} / 3$. Thus, we can define the maps $P_{u}: \mathcal{B}_{\delta_{0} / 3}(w) \cap \Lambda \rightarrow W_{\eta}^{u}(x) \cap \Lambda$ and $P_{s}: \mathcal{B}_{\delta_{0} / 3}(w) \cap \Lambda \rightarrow W_{\eta}^{s}(x) \cap \Lambda$ by $P_{u}(v):=[v, x]$ and $P_{s}(v):=[x, v]$, for $v \in \mathcal{B}_{\delta_{0} / 3}(w)$. These maps are clearly continuous. Observe that, given $v \in \mathcal{B}_{\delta_{0} / 3}(w) \cap \Lambda$, we have that $d(x, v)<d(x, w)+d(w, v)<\delta_{0} / 3+\delta_{0} / 3<\delta_{0}$. Given that $w=[y, z]$, we have $P_{u}(w)=y$ and $P_{s}(w)=z$, by expansivity on $\Lambda$. Hence, there is a neighborhood $U \subset \mathcal{B}_{\delta_{0} / 3}(w) \cap \Lambda$ of $w$ in $\Lambda$ such that $P_{u}(U) \subset V_{\delta_{1}}^{u}(x)$ and $P_{s}(U) \subset V_{\delta_{1}}^{s}(x)$. Take $v \in U$. By expansivity on $\Lambda$, we have that $v=[[v, x],[x, v]]$. Therefore, $v \in\left[V_{\delta_{1}}^{u}(x), V_{\delta_{1}}^{s}(x)\right]$, which proves that $\left[V_{\delta_{1}}^{u}(x), V_{\delta_{1}}^{s}(x)\right]$ is an open set of $\Lambda$. Thus, $\mathrm{B}(\mathrm{i})$ is proved.

To prove B(ii), define a map $h:\left[V_{\delta_{1}}^{u}(x), V_{\delta_{1}}^{s}(x)\right] \rightarrow V_{\delta_{1}}^{u}(x) \times V_{\delta_{1}}^{s}(x)$ by $h(w):=([w, x],[x, w])$, for $w \in\left[V_{\delta_{1}}^{u}(x), V_{\delta_{1}}^{s}(x)\right]$. Clearly, $h$ is continuous and $h$ itself is the inverse map of $[\cdot, \cdot]$.

Finally we shall prove B(iii). Define the map $g_{2}: \Delta\left(\delta_{0}\right) \rightarrow \mathbb{R}$ by $g_{2}(x, y):=\operatorname{diam}\{x,[y, x],[x, y]\}$, for $(x, y) \in \Delta\left(\delta_{0}\right)$. Then $g_{2}$ is (uniformly) continuous and, therefore, there exists $\rho \in\left(0, \delta_{1}\right)$ such that $d(x, y)<\rho$ implies $g_{2}(x, y)<\delta_{1}$. Hence, $[y, x] \in V_{\delta_{1}}^{u}(x)$ and $[x, y] \in V_{\delta_{1}}^{s}(x)$. Therefore, given $y \in \Lambda$ with $d(x, y)<\rho$, we have that $y=[[y, x],[x, y]] \in\left[V_{\delta_{1}}^{u}(x), V_{\delta_{1}}^{s}(x)\right]$, which proves B(iii).

\section{TOPOLOGICAL HOMOCLINIC CLASSES}

3.1. Topological transversality. The intersection between two curves in the plane can be quite odd. Indeed, given any compact set $K \subset \mathbb{R}$, let us define the continuous function $f: \mathbb{R} \rightarrow \mathbb{R}$ by $f(x)=\min \{|x-k|: k \in K\}$. The intersection between the 1-dimensional manifolds defined by the graph of $f$ and the $x$-axis is the set $(k, 0) \in \mathbb{R}^{2}$ where $k \in K$. Let $B(0, r) \subset \mathbb{R}^{2}$ stands for the open ball centered in $(0,0)$ and with radius $r$. Given 1-dimensional manifolds $N_{1}, N_{2} \subset \mathbb{R}^{2}$, we say that $q \in \mathbb{R}^{2}$ is a transversal intersection between $N_{1}$ and $N_{2}$ if there exist an open ball $B$ containing $q$ and a homeomorphism $h: B(0, r) \rightarrow B$ where $r>0$ such that $h(x, 0)=\hat{N}_{1}$ and $h(0, y)=\hat{N}_{2}$, where $\hat{N}_{i}$ are the connected components of $N_{i} \cap B$ containing $q$.

In the sequel we apply the previous concept of transversality to stable and unstable topological manifolds. Clearly, if $q \in W^{s}(p) \cap W^{u}(p)$ is a transversal intersection between the stable and unstable manifold of $p$, then this intersection is persistent in the sense that the intersection between these two 1-dimensional manifolds cannot disappear under an arbitrarily small $C^{0}$-perturbation of the map. We denote that $q \in W^{s}(p) \cap W^{u}(p)$ is a transversal intersection by writing $q \in W^{s}(p) \pitchfork$ $W^{u}(p)$. 
3.2. Definition of topological homoclinic classes. Let $f: M \rightarrow M$ be an area-preserving homeomorphism and $p \in M$ a periodic point of period $n$.

We say that $p$ is topologically hyperbolic if $f$ is a hyperbolic homeomorphism on $\Lambda=O(p)$, the orbit of $p$.

By Proposition 2.1, under the expansiveness hypothesis on $\Lambda$, stable and unstable sets of points $x \in \Lambda$ are dynamically defined. Clearly, by Remark 2.1 any stable/unstable structure at topological hyperbolic periodic points must be a 2-prongs structure.

We define the topological homoclinic class of a topological hyperbolic periodic point $p$ by the closure of the transversal intersections of the stable and unstable manifolds of $f^{i}(p)$, for $i=$ $0, \ldots, n-1$, that is by $\overline{W^{s}\left(f^{i}(p)\right) \pitchfork W^{u}\left(f^{i}(p)\right)}$, for $i=0, \ldots, n-1$. Clearly, a homoclinic class is compact and $f$-invariant. The compactness follows from the fact that it is a closed subset of a compact manifold. To check that $f(\Lambda)=\Lambda$, observe that if we have $x \in \Lambda$, then $x_{n} \rightarrow x$ for a sequence $\left\{x_{n}\right\}_{n} \subset W^{s}(p) \cap W^{u}(p)$. By the $f$-invariance of the sets $W^{s}(p)$ and $W^{u}(p)$ we get that $\left\{f\left(x_{n}\right)\right\}_{n} \subset W^{s}(p) \cap W^{u}(p)$. Finally, the continuity of $f$ ensures that $f(x) \in \Lambda$.

3.3. The Birkhoff-Smale theorem. In Theorem 3.2 we will obtain a slightly different version of the well-known Birkhoff-Smale theorem but with a topological flavor. The fixed point index will play a crucial role along the proof since, in rough terms, the existence of non-null index on a set assures a fixed point in that set. Let us recall the definition of fixed point index: take an open ball $B \subset M$ such that Fix $(f, \partial B)=\emptyset$ (meaning that $f$ has no fixed points in the boundary of $B$ denoted by $\partial B$ ) and $\bar{B} \cap \overline{f(B)} \neq \emptyset$ and $\partial B, \bar{B} \cup \overline{f(B)}$ are labelled by the same chart. In this case we say that the index of $f$ in $B$ is the degree of the map defined in chart coordinates by the vector field:

$$
\begin{aligned}
X_{f}: \quad \partial B \simeq \mathbb{S}^{1} & \longrightarrow \frac{\mathbb{S}^{1}}{x} \\
x & \longmapsto \frac{f(x)-x}{\|f(x)-x\|}
\end{aligned}
$$

If the index is $\neq 0$, then $f$ has a fixed point in $B$. When $\bar{B} \cap \overline{f(B)}=\emptyset$ we say that the index is zero.

In the proof of Theorem 3.2 we will need a weak version of the well-known Lambda-lemma ([7]). This lemma tells us that if $\Sigma$ is a section transversal to the stable manifold, then $f^{n}(\Sigma)(n>0)$ becomes arbitrarily $C^{1}$ close to some compact subset contained in the unstable manifold. The difficult part in the proof of the classical Lambda-Lemma is to obtain the $C^{1}$ closeness of the two sets. Indeed, we can obtain easily the next:

Lemma 3.1. (Topological Lambda-Lemma) Let $\Sigma$ and $W^{s}(p)$ be transversal topological manifolds and $\Gamma \subset W^{u}(p)$ be a compact set. Then, given any $\epsilon>0$, there exist $\Sigma^{\prime} \subset \Sigma$ and $n_{0} \in \mathbb{N}$ such that $d_{H}\left(f^{n_{0}}\left(\Sigma^{\prime}\right), \Gamma\right)<\epsilon$ (where $d_{H}$ stands for the Hausdorff distance between sets).

Now we are ready to state the main result in this section.

Theorem 3.2. (Topological Birkhoff-Smale theorem) Let $M$ be a surface, $f: M \rightarrow M$ a homeomorphism, $p \in M$ a periodic topological hyperbolic point of period $n$ and $q \in W^{s}(p) \pitchfork W^{u}(p)$. Then $f$ has a periodic point in any neighborhood of $q$.

Proof. Let $q \in W^{s}(p) \pitchfork W^{u}(p)$ and let $\Gamma \subset W^{u}(p)$ be the compact arc with extremes $p$ and $q$. By hypothesis there exists a section $\Sigma$ which is transversal to $W^{s}(p)$ and containing $q$. Then, by Lemma 3.1, given any $\epsilon>0$, there exist $\Sigma^{\prime} \subset \Sigma$ and $n_{0} \in \mathbb{N}$ such that $d_{H}\left(f^{n_{0}}\left(\Sigma^{\prime}\right), \Gamma\right)<\epsilon$. Take a rectangle $R$ like in Figure 4 sufficiently thin and sufficiently close to $W^{u}(p)$ in order to contain $\Sigma^{\prime}$. We obtain $R \cap f^{n_{0}}(R) \neq \emptyset$. Moreover, we can even assure that $\operatorname{Fix}\left(f^{n_{0}}, \partial R\right)=\emptyset$. Indeed, the 


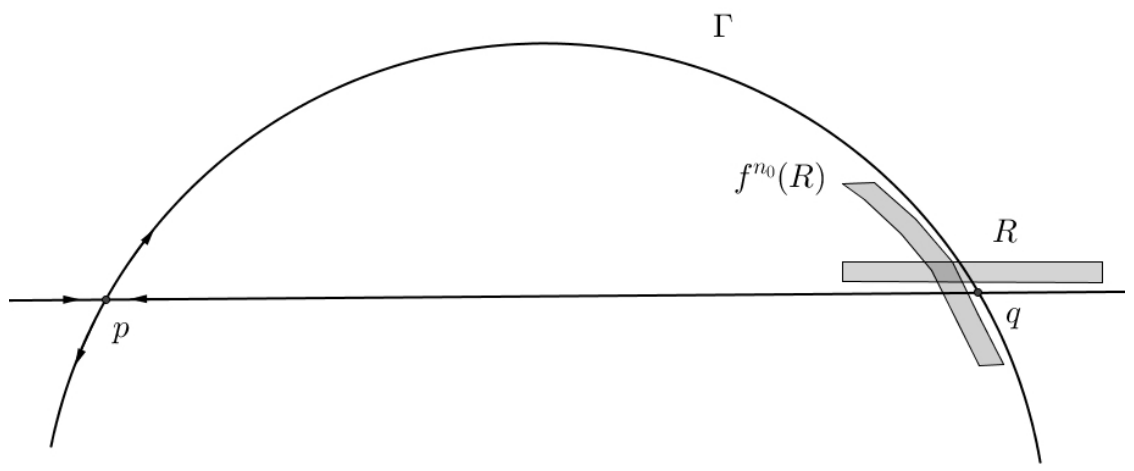

FIGURE 4. Support to the proof of Theorem 3.2.

top and the bottom of $R$, in a configuration like in Figure 4, turn into the bottom and the top of $f^{n_{0}}(R)$ respectively. Of course that a configuration like in Figure 4 can be achieved by allowing the change of $R$ a little bit. Since $\partial R$ is homeomorphic to $\mathbb{S}^{1}$ we let $X_{f^{n}}$ be a vector field defined in $\partial R$ as above. The angular variation of $X_{f^{n_{0}}}$ as $x$ moves once around $\partial R$ is not zero, then $f^{n_{0}}$ has a fixed point in $R$. Consequently, $f$ has a periodic point in any neighborhood of $q$.

Remark 3.1. We point out that the classic Birkhoff-Smale theorem ([11]) ensures that the periodic points in any neighborhood of $q$ are homoclinic related with $p$. This allows us to conclude that the periodic orbits in the homoclinic class are dense in the homoclinic class. However, the arguments used in [11] are clearly not valid outside the differentiable context. An alternative approach using tubular family theorems ( $c f$. [8]) is also not suitable under $C^{0}$ hypothesis.

\section{Proof of Theorem 1}

Let $M$ be a surface, $f: M \rightarrow M$ be an area-preserving homeomorphism and let $\Lambda \subseteq M$ be a topological homoclinic class of $f$. We already know by Corollary 2.5 that, if $f$ is a hyperbolic homeomorphism on $\Lambda$ with expansive constant $e$, then for each $0<\epsilon \leq e / 2$ there exists $\delta>0$ such that if $x, y \in \Lambda$ and $d(x, y)<\delta$, then

$$
W_{\epsilon}^{s}(x) \cap W_{\epsilon}^{u}(y)=\{\text { one point }\} \subset \Lambda .
$$

So, if $\Lambda$ is a topological homoclinic class displaying shadowing and expansiveness with expansive constant $e$, then for each $0<\epsilon \leq e / 2$ there exists $\delta>0$ such that if $x, y \in \Lambda$ and $d(x, y)<\delta$, then (4.1) holds. Since $\Lambda$ is a homoclinic class it is closed by definition. As $M$ is connected it is sufficient to prove that $\Lambda$ is open in $M$.

The next result will be central in the proof of Theorem 1 .

Lemma 4.1. If $x \in \Lambda$ then $\Lambda \cap W^{u}(x)$ is dense in $W^{u}(x)$ and $\Lambda \cap W^{s}(x)$ is dense in $W^{s}(x)$.

Proof. The proof is by contradiction. We assume that $\Lambda \cap W^{u}(x)$ is not dense in $W^{u}(x)$. Denote by $I \subset W^{u}(x)$ a gap interval in $W^{u}(x) \backslash \Lambda$ that is, its extremes are in $\Lambda$ when $I$ is bounded (from one side, from the other or both). 
Case 1: We begin by considering that $I=\left[y_{1}, y_{2}\right]$ with $y_{1}, y_{2} \in \Lambda$. In $\S 2.3$ we considered small constants like $\epsilon, \delta_{0}, \delta_{1}$ and $\rho$ because the arguments involving the LPS hold only in a microscopic level. Therefore, we iterate backward $I$ to be sufficiently close to $x$ and so be under the hypotheses of $\S 2.3$. Clearly, an iterate of $I$ will still be a gap interval. Using Proposition 2.6, we consider a periodic point $z \in \Lambda$ arbitrarily close to $x$ and such that $W^{u}(O(x)) \cap W^{u}(O(z))=\emptyset$. Since $y_{1}, y_{2} \in \Lambda$, by Corollary 2.5, we obtain that $W_{\epsilon}^{s}\left(y_{1}\right)$ intersects $W_{\epsilon}^{u}(z)$ in a single point $x_{1} \in \Lambda$ and $W_{\epsilon}^{s}\left(y_{2}\right)$ intersects $W_{\epsilon}^{u}(z)$ in a single point $x_{2} \in \Lambda$. Let $R$ be the rectangle with sides $I$, the segment $I_{1}$ with extremes $y_{1}$ and $x_{1}$, the segment $I_{2}$ with extremes $y_{2}$ and $x_{2}$ and the segment $I_{3}$ with extremes $x_{1}$ and $x_{2}$. To assure that $R \cap \Lambda=\emptyset$ we can go up in the proof and pick other periodic point $z$ closer to $x$ building another rectangle $R$ satisfying $R \cap \Lambda=\emptyset$. Indeed, if such $z$ do not exists, then there would a point $w \in R \cap \Lambda$ such that $W_{\epsilon}^{s}(w) \pitchfork W_{\epsilon}^{u}(x) \in I$ which contradicts the fact that $I$ is a gap interval. From Poincaré's recurrence theorem $\lambda$-almost every point in $R$ is recurrent and so $f^{n}(R) \cap R \neq \emptyset$ for infinitely many choices of $n$. Moreover, since $f$ is an area-preserving map, we obtain $\partial R \cap \partial f^{n}(R) \neq \emptyset$. In fact, preservation of area avoids $R \subset f^{n}(R)$ or $R \supset f^{n}(R)$ and so the intersection of boundaries is inevitable. Furthermore, from stable/unstable sets arguments we get $f^{n}(I) \cap I=\emptyset, f^{n}(I) \cap I_{3}=\emptyset, f^{n}\left(I_{3}\right) \cap I=\emptyset$, $f^{n}\left(I_{3}\right) \cap I_{3}=\emptyset, f^{n}\left(I_{1}\right) \cap I_{1}=\emptyset, f^{n}\left(I_{1}\right) \cap I_{2}=\emptyset, f^{n}\left(I_{2}\right) \cap I_{2}=\emptyset$ and $f^{n}\left(I_{2}\right) \cap I_{1}=\emptyset$. Therefore, we must have an intersection between $f^{n}$ iterates of the segments $I, I_{3}$ with the segments $I_{1}, I_{2}$, i.e., $f^{n}\left(I \cup I_{3}\right) \cap\left(I_{1} \cup I_{2}\right) \neq \emptyset$, contradicting the fact that $I$ was a gap interval.

Case 2: Finally, we consider the case of having an unbounded interval $I=\left[y_{1},+\infty\right) \subset W^{u}(x)$ with $y_{1} \in \Lambda$. Once again we pull $I$ near $x$. Clearly, an iterate of $I$ will still be an unbounded interval. We take a periodic point $z \in \Lambda$ arbitrarily close to $x$. Since $z \in \Lambda$, by Corollary 2.5, we get that $W_{\epsilon}^{u}(z) \cap W_{\epsilon}^{s}(x) \neq \emptyset$ and $W_{\epsilon}^{u}(z) \cap W_{\epsilon}^{s}\left(y_{1}\right) \neq \emptyset$ and, moreover, each of these intersections is a single point in the topological homoclinic class $\Lambda$.

Therefore, in $W^{u}(z)$ and since $z$ is periodic, if exists, a gap interval cannot be unbounded and we can apply Case 1 and conclude that $W^{u}(z) \cap \Lambda$ is dense in $W^{u}(z)$. Since, by Proposition 2.6, we can take periodic points $z_{n} \in \Lambda$ such that $W^{u}\left(z_{n}\right) \cap \Lambda$ is dense in $W^{u}\left(z_{n}\right)$, we obtain that $I \cap \Lambda \neq \emptyset$ which is a contradiction.

Finally we prove that $\Lambda$ is open in $M$. By Lemma $2.7, f$ has a local product structure on $\Lambda$. Consider $\delta_{0}>0$ and $\delta_{1} \in\left(0, \delta_{0} / 2\right)$ given by the local product structure on $\Lambda$. We have that $\left[V_{\delta_{1}}^{u}(x), V_{\delta_{1}}^{s}(x)\right]$ is an open set of $\Lambda$ with diameter less than $\delta_{0}$ and also $[\cdot, \cdot]: V_{\delta_{1}}^{u}(x) \times V_{\delta_{1}}^{s}(x) \rightarrow$ $\left[V_{\delta_{1}}^{u}(x), V_{\delta_{1}}^{s}(x)\right]$ is a homeomorphism.

By Lemma 4.1 the set $V_{\delta_{1}}^{u}(x)$ is dense in $W_{\delta_{1}}^{u}(x)$ and the set $V_{\delta_{1}}^{s}(x)$ is dense in $W_{\delta_{1}}^{s}(x)$. Clearly, the closure of the Cartesian product $V_{\delta_{1}}^{u}(x) \times V_{\delta_{1}}^{s}(x)$ is a topological disk and a neighborhood of $x$. Since $[\cdot, \cdot]: V_{\delta_{1}}^{u}(x) \times V_{\delta_{1}}^{s}(x) \rightarrow\left[V_{\delta_{1}}^{u}(x), V_{\delta_{1}}^{s}(x)\right]$ is a homeomorphism, then $[\cdot, \cdot]\left(\overline{V_{\delta_{1}}^{u}(x) \times V_{\delta_{1}}^{s}(x)}\right)=$ $\overline{\left[V_{\delta_{1}}^{u}(x), V_{\delta_{1}}^{s}(x)\right]}$. Hence, $x$ is in the interior of $\Lambda$ in $M$. Thus, $\Lambda$ is open in $M$.

\section{ACKNOWLEDGEMENTS}

MB was partially supported by FCT - 'Fundação para a Ciência e a Tecnologia', through Centro de Matemática e Aplicações (CMA-UBI), Universidade da Beira Interior, project

UID/MAT/00212/2013. MB would like to thanks CMUP for providing the necessary conditions in which this work was also developed. 
MJT was partially supported by the Research Centre of Mathematics of the University of Minho with the Portuguese Funds from the 'Fundação para a Ciência e a Tecnologia', through the Project UID/MAT/00013/2013.

\section{REFERENCES}

[1] E. Akin, M. Hurley and J. Kennedy, Dynamics of Topologically Generic Homeomorphisms, Memoirs of the American Mathematical Society, Volume 164, number 783, 2003.

[2] N. Aoki, Topological Dynamics, in Topics in General Topology, Edited by Kiiti Morita and Jun-iti Nagata, North-Holland Mathematical Library, Volume 41, 1989, Chapter 15, 625-740.

[3] K. Hiraide, Expansive homeomorphisms of compact surfaces are pseudo-Anosov, Osaka J. Math 27 (1990), 117-162.

[4] J. Lewowicz, Expansive Homemorphisms of Surfaces, Bol. Soc. Bras. de Mat., 20 (1989), 113-133.

[5] S. Newhouse, Hyperbolic Limit Sets, Trans. Amer. Math. Soc., 167 (1972), 125-150.

[6] S. Newhouse, Topics in Conservative Dynamics. Regular and Chaotic Motions in Dynamic Systems, Edited by G. Velo and A. S. Wightman. D. Reidel Publishing Company, Dordrecht-Holland. NATO Advanced Study Institutes Series, Volume 118, 1985, 103-184.

[7] J. Palis and W. de Melo, Geometric theory of dynamical systems. An introduction, Springer-Verlag, New York-Berlin, 1982.

[8] J. Palis and S. Smale, Structural stability theorems, Proc. Sympos. Pure Math., vol. 14, Amer. Math. Soc, Providence, R. I., 1970.

[9] M. Shub, Global Stability of Dynamical Systems, Springer-Verlag, 1987.

[10] S. Smale, Differentiable dynamical systems, Bull. A.M.S., 73 (1967), 747-817.

[11] S. Smale, Diffeomorphisms with many periodic points, Differential and Combinatorial Topology (A Symposium in Honor of Marston Morse), Princeton Univ. Press, Princeton, N. J., 1965, pp. 63-80.

Departamento de Matemática, Universidade da Beira Interior, Rua Marquês d’Ávila e Bolama, 6201-001 CovILhã, Portugal.

Email address: bessa@ubi.pt

CMat and Departamento de Matemática e Aplicações, Universidade do Minho, Campus de Gualtar, $4700-057$ Braga, Portugal

Email address: jtorres@math.uminho.pt 